\title{
ON CYCLIC MAPS
}

\author{
K. L. LIM \\ (Received 3 December 1980) \\ Communicated by J. H. Rubinstein
}

\begin{abstract}
The definition of cyclic maps is recalled and their existence discussed. Among other things, it is shown that cyclicity of maps is closed under product and that if $f$ is cyclic then $\Omega f$ is central. Some results of Gottlieb (1972) on homology are applied to investigate the relationship between cyclicity of maps and maps of finite order.
\end{abstract}

1980 Mathematics subject classification (Amer. Math. Soc.): 55 E 05.

\section{Introduction}

Let $X$ be a topological space and $I$ the closed unit interval $[0,1]$. A cyclic homotopy (see Ganea (1968) and Gottlieb (1965)) $H: X \times I \rightarrow X$ is a homotopy such that $H(x, 0)=H(x, 1)=x$ for all $x$ in $H$. If $H$ is a cyclic homotopy, then the path $\sigma:$ $I \rightarrow X$ given by $\sigma(t)=H(*, t)$ for all $t$ in $I$, is called the trace of $H$. Gottlieb (1965) introduced the subgroup $G(X)$ (of the fundamental group) which consists of all the homotopy classes of those loops which are the trace of some cyclic homotopy. Later, Varadarajan (1969) generalized $G(X)$ to $G(A, X)$ for any space $A$ and called the maps $f: A \rightarrow X$ which are represented by elements of $G(A, X)$ "cyclic". We shall follow this convention (see also Hoo (1972)). In Section 2, some concepts and notations which will be used later are recalled. Section 3 is devoted to the definition and existence of cyclic maps. In Section 4, we show, among other things, that cyclicity of maps is closed under product and that if $f$ is cyclic then $\Omega f$ is central. Some results of Gottlieb (1972) on homology are applied in Section 5 to investigate the relationship between cyclicity of maps and maps of finite order.

(c) Copyright Australian Mathematical Society 1982 


\section{Preliminaries}

We shall now establish some notations and terminologies that will be used throughout this paper. Unless otherwise stated, we shall work in the category of spaces with base points and having the homotopy type of locally finite $\mathrm{CW}$ complexes. All maps shall mean continuous functions. All homotopies and maps are to respect base points. The base point as well as the constant map will be denoted by *. 1 (sometimes with decoration) will denote the identity function (resp. map) of a set or a group (resp. space) when it is clear from the context. For simplicity, we sometimes use the same symbol for a map and its homotopy class.

All function spaces will be endowed with the compact-open topology and, unless otherwise stated, the constant map will be taken to be the base point. $X^{X}$ shall denote the space of free maps from $X$ to $X$ with $1_{X}$ as base point. The evaluation map $\omega: X^{X} \rightarrow X$ is defined to be $\omega(f)=f(*)$ for each $f \in X^{X}$.

The diagonal map $\Delta: X \rightarrow X \times X$ is given by $\Delta(x)=(x, x)$ for each $x \in X$, the folding map $\nabla: X \vee X \rightarrow X$ by $\nabla(x, *)=\nabla(*, x)=x$ for each $x \in X$, and the switching map $T: X \times Y \rightarrow Y \times X$ by $T(x, y)=(y, x)$ for each $x \in X, y \in Y$.

Frequently (not always) $i$ and $j$ will be reserved for the inclusion maps of the form $i_{1}: X \rightarrow X \times Y$ or $i_{2}: Y \rightarrow X \times Y$, and $j: X \vee Y \rightarrow X \times Y$ respectively. The projection is denoted by $p$ with decoration.

$\Sigma X$ and $\Omega X$ denote the reduced suspension and the loop space of $X$ respectively. The adjoint functor from the group $[\Sigma X, Y]$ to the group $[X, \Omega Y]$ will be denoted by $\tau$. The symbols $e_{A}$ and $e_{A}^{\prime}$ denote $\tau^{-1}\left(l_{\Omega A}\right)$ and $\tau\left(l_{\Sigma A}\right)$ respectively, the subscript will be dropped if there is no danger of confusion.

Let $n>1$ be an integer. A space $X$ is said to be $(n-1)$-connected iff $\pi_{k}(X)=0$ for all $k \leqslant n-1$.

The following well-known results are frequently used:

(1) If $A$ is a co- $H$-space, then we can find a map $s: A \rightarrow \Sigma \Omega A$ such that $e s \simeq 1_{A}$.

(2) If $B$ is an $H$-space, then we can find a map $s^{\prime}: \Omega \Sigma B \rightarrow B$ such that $s^{\prime} e^{\prime} \simeq 1_{B}$.

(3) Let $A$ and $B$ be an $H$-cogroup and an $H$-group respectively. Then [ $A, X]$ and $[X, B]$ are groups for any space $X$.

\section{Definition and existence of cyclic maps}

Definition 3.1. A map $f: A \rightarrow X$ is said to be cyclic if there exists a map $F$ : $X \times A \rightarrow X$ such that the following diagram is homotopy commutative: that is, $F j \simeq \nabla(1 \vee f)$. Since $j$ is a cofibration, this is equivalent to saying that we can find a map $G: X \times A \rightarrow X$ such that $G j=\nabla(1 \vee f)$. We call such a map $G$ an 
associated map of $f$. The set of all homotopy classes of cyclic maps from $A$ to $X$ is denoted by $G(A, X)$ and is called the Gottlieb subset of $[A, X]$

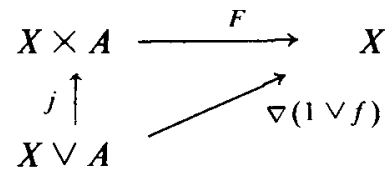

REMARK 1. Note that a map $f: A \rightarrow X$ is cyclic if and only if there exists a map $F: X \times A \rightarrow X$ such that $F i_{1}=1_{X}$ and $F i_{2}=f$, where $i_{1}: X \rightarrow X \times A$ and $i_{2}$ : $A \rightarrow X \times A$ are inclusions, that is, $F$ is of type $(1, f)$. Clearly the constant map *: $A \rightarrow X$ is cyclic.

REMARK 2. If $A=S^{n}$ ( $n \geqslant 1$ is an integer), then $G(A, X)$ reduces to $G(X)$ (Gottlieb (1965)) and $G_{n}(X)$ (Gottlieb (1969)) which is called the $n$th evaluation subgroup of $X$.

LeMma 3.2 (Varadarajan (1969)). Let $f: A \rightarrow X$ be a cyclic map and $\theta: B \rightarrow A$ an arbitrary map. Then $f \theta: B \rightarrow X$ is a cyclic map.

The existence of cyclic maps is easily seen from the previous remark and the following proposition.

Proposition 3.3. Let $X$ be a space. Then the following are equivalent:

(i) $X$ is an $H$-space.

(ii) $1_{x}$ is cyclic.

(iii) $G(A, X)=[A, X]$ for any space $A$.

Proof. (i) $\Rightarrow$ (ii). Let $m$ be the $H$-structure on $X$. Then $m j \simeq \nabla=\nabla\left(1_{X} \vee 1_{X}\right)$, so that $1_{X}$ is cyclic.

(ii) $\Rightarrow$ (iii). Let $A$ be any space and let $f \in[A, X]$. Then $f=1_{X} f$ is cyclic, by Lemma 3.2.

(iii) $\Rightarrow$ (i). Take $A=X$. Then $1_{X}$ is cyclic, so that we can find a map $m$ : $X \times X \rightarrow X$ such that $m j=\nabla$.

Another way in which cyclic maps arise naturally is by fibrations. Suppose $F \rightarrow E \rightarrow B$ is a fibration. Then we have an operation $\rho: F \times \Omega B \rightarrow F$ of the loop space of the base on the fibre. The fibration gives rise to a Puppe sequence $\cdots \rightarrow \Omega E \rightarrow \Omega B \stackrel{\partial}{\rightarrow} F \rightarrow E \rightarrow B$. We can take $\partial=\rho \mid \Omega B$, that is, $\rho$ is a map of type $(1, \partial)$, or $\partial$ is cyclic. It follows that for all spaces $A, \partial_{\sharp}[A, \Omega B] \subset G(A, F)$. If 
$G(A, F)=0$, then we can obtain some information on the fibration. More precisely, we have the following result.

THEOREM 3.4. Let $F \rightarrow E \stackrel{p}{\rightarrow} \Sigma A$ be a fibration. If $G(A, F)=0$, then $p$ has a cross-section.

Proof. If $G(A, F)=0$, then $\partial_{\sharp}[A, \Omega \Sigma A]=0$. Hence from the exact sequence of the fibration, we see that $(\Omega p)_{\sharp}:[A, \Omega E] \rightarrow[A, \Omega \Sigma A]$ is onto. In particular, we can find a map $f: A \rightarrow \Omega E$ such that $(\Omega p) f \simeq e^{\prime}$, where $e^{\prime}: A \rightarrow \Omega \Sigma A$ is the adjoint of the identity map $\Sigma A \rightarrow \Sigma A$. Taking adjoints, we obtain $p \tau^{-1}(f) \simeq 1_{\Sigma A}$, where $\tau^{-1}(f)$ is the adjoint of $f$. Thus we obtain a cross-section.

EXAMPLE 3.5. Any fibration $E \rightarrow S^{2 n+1}$ with fibre $S^{2 n}(n \geqslant 1)$ admits a cross-section. In fact, $G_{2 n}\left(S^{2 n}\right)=0$, by Theorem 5.4 of Gottlieb (1969).

A third way of getting cyclic maps is as follows: Let $G$ be a topological group and let $H$ be a closed subgroup. Let $G / H$ denote the space of left cosets and let $p$ : $G \rightarrow G / H$ be the natural map. Then $p$ is cyclic since we have a natural map $G / H \times G \rightarrow G / H$ given by $\left(g_{1} H, g_{2}\right) \rightarrow g_{2} g_{1} H$, of type $(1, p)$. For further detail about this, see Lang (1970).

\section{Some basic properties of cyclic maps}

Let $\theta: B \rightarrow A$ and $g: X \rightarrow Y$ be maps such that $g$ has a right homotopy inverse. Then if $f: A \rightarrow X$ is cyclic, so are $f \theta$ and $g f$.

Example 4.1. Let $A$ be a co- $H$-space and $f: A \rightarrow X$ a map. Then $f$ is cyclic if and only if $f e: \Sigma \Omega A \rightarrow X$ is cyclic. In fact, there exists a map $s: A \rightarrow \Sigma \Omega A$ such that $e s \simeq 1_{A}$, so that $f \simeq f e s$.

EXAMPLE 4.2. Let $\alpha \in \pi_{2 n+1}(X)$ be such that any representative $f$ of $\alpha$ has a right homotopy inverse, then $2 \alpha \in G_{2 n+1}(X)$. For if $f: S^{2 n+1} \rightarrow S^{2 n+1}$ is a map of degree 2, then $2[g]=[g f]$ and $f$ is cyclic, by Theorem 5.4 of Gottlieb (1969). Thus $g f$ is cyclic.

Definition 4.3 (Hu (1959)). A space $X$ is said to be $m$-coconnected $(m \geqslant 1$ is an integer) if $H^{q}(X ; G)=0$ for each $q \geqslant m$ and for each coefficient group $G$. 
Lemma 4.4 (Hu (1959), page 213). Let $X$ and $Y$ be two $(2 m-1)$-coconnected spaces. If $f: X \rightarrow Y$ is a map, then $f^{\#:} \pi^{m}(Y) \rightarrow \pi^{m}(X)$ is a homomorphism, where $\pi^{m}(X)$ and $\pi^{m}(Y)$ are the mth cohomotopy groups of $X$ and $Y$ respectively.

Proposition 4.5. Let $B$ be $(4 n+1)$-coconnected. If $g: B \rightarrow S^{2 n+1}$ is any map, then $2 \mathrm{~g}$ is cyclic.

Proof. If $n=0$, then $\left[B, S^{1}\right]=G\left(B, S^{1}\right)$ since $S^{1}$ is an $H$-space. Thus the proposition is true for $n=0$.

Assume $n>0$. Then $S^{2 n+1}$ is $(4 n+1)$-coconnected. Let $f: S^{2 n+1} \rightarrow S^{2 n+1}$ be a map of degree 2 and $\iota$ a generator of $\pi^{2 n+1}\left(S^{2 n+1}\right)=\pi_{2 n+1}\left(S^{2 n+1}\right)=Z$. According to Lemma 4.4, $g^{\#:} \pi^{2 n+1}\left(S^{2 n+1}\right) \rightarrow \pi^{2 n+1}(B)$ is a homomorphism since $B$ and $S^{2 n+1}$ are $(4 n+1)$-coconnected. Hence $g^{\sharp}(f)=g^{\sharp}(2 \iota)=g^{\sharp}(\iota+\iota)=g^{\sharp}(\iota)+$ $g^{\sharp}(\iota)=g+g=2 g$, so that $f g=2 g$. Now since $f$ is cyclic, it follows that $2 g$ is also cyclic.

The next result says that cyclicity of maps is closed under product.

Proposition 4.6. If the maps $f: A \rightarrow X$ and $g: B \rightarrow Y$ are cyclic, then so is $f \times g$ : $A \times B \rightarrow X \times Y$.

Proof. Let $F$ and $G$ be two associated maps of $f$ and $g$ respectively. Let $H=(F \times G)(1 \times T \times 1):(X \times Y) \times(A \times B) \rightarrow X \times Y$. Then $H$ is an associated map of $f \times g$.

It might be supposed that if $f: A \rightarrow X$ and $g: B \rightarrow Y$ are cyclic then so is $f \vee g$ : $A \vee B \rightarrow X \vee Y$. That this is not true can be illustrated by the following example.

Example 4.7. Let $A=B=X=Y=S^{1}$. Then $1_{S^{1}}$ is cyclic. But $1_{S^{1}} \vee 1_{S^{1}}=$ $1_{S^{\prime} \vee S^{\prime}}$ is not cyclic by Proposition 3.3, for $S^{1} \vee S^{1}$ is not an $H$-space.

Lemma 4.8. If the maps $f: A \rightarrow X$ and $g: B \rightarrow X$ are cyclic, then so is $\nabla(f \vee g)$ : $A \vee B \rightarrow X$.

Proof. Let $F$ and $G$ be two associated maps of $f$ and $g$ respectively. Let $h$ : $X \times(A \times B) \rightarrow(X \times A) \times B$ be the homeomorphism. Let $K \equiv G\left(F \times 1_{B}\right) h$ : $X \times(A \times B) \rightarrow X$ and $H \equiv K \mid X \times(A \vee B) \rightarrow X$. Then $H$ is an associated map of $\nabla(f \vee g)$.

The next corollary is an immediate consequence of Lemmas 3.2 and 3.3. 
CoRollary 4.9. If $A$ is a co-H-space, then $G(A, X) \subset[A, X]$ is closed under the natural operation induced by the co-H-structure on $A$.

We recall the following definition and results:

Definition 4.10 (Arkowitz and Curjel (1967)). Let $(G, m, \mu)$ be an $H$-group and $A$ any space. We say that a map $f: A \rightarrow G$ is central if $c(1 \times f) \simeq *$ where $c$ : $G \times G \rightarrow G$ is the basic commutator map (that is, $c \equiv m(m \times m)(1 \times 1 \times \mu \times$ $\mu) \Delta)$.

Lemma 4.11 (Arkowitz and Curjel (1967)). (i) Let $p_{1}: G \times A \rightarrow G$ and $p_{2}$ : $G \times A \rightarrow A$ be the projections. Then $f$ is central if and only if the commutator $\left(p_{1}, f p_{2}\right) \equiv p_{1}+f p_{2}-p_{1}-f p_{2} \simeq *$ in $[G \times A, G]$.

(ii) Any central map $f: A \rightarrow G$ lies in the center of $[A, G]$.

(iii) Let $f: A \rightarrow G$ be central and $\theta: B \rightarrow A$ an arbitrary map. Then $f \theta: B \rightarrow G$ is central.

Let $f: A \rightarrow X$ be a map. It is evident from the preceding lemma that if $\Omega f$ is central, then $(\Omega f)_{\sharp}:[Z, \Omega A] \rightarrow[Z, \Omega X]$ has image contained in the center of $[Z, \Omega X]$ for any space $Z$.

The following lemma is due to Ganea:

LEMMA 4.12 (Ganea (1967)). Let $X b A \stackrel{L}{\rightarrow} X \vee A \rightarrow X \times A$ be a fibration. Then $\nabla(l \vee f) L=*$ if and only if $\Omega f$ is central.

Proposition 4.13. If $f$ is cyclic, then $\Omega f$ is central.

Proof. Since $f$ is cyclic, $\nabla(1 \vee f)$ extends to a map $X \times A \rightarrow X$, so that $\nabla(1 \vee f) L \simeq *$ and the assertion follows from the preceding lemma.

\section{Cyclicity of maps and maps of finite order}

In this section we make some further observations on cyclic maps using some results of Gottlieb (1972) on homology. Following Gottlieb (1972), we observe that composition of maps makes $X^{X}$ an $H$-space with $1_{X}$ as base point, where $X^{X}$ is the space of free maps from $X$ to $X$. If $\mu$ is the composition map, then we can define a multiplication on $H_{*}\left(X^{X} ; Q\right)$ by $x y=\mu_{*}(x \otimes y)$ for all $x, y$ in $H_{*}\left(X^{X} ; Q\right)$, where $Q$ is the field of rationals. With the diagonal map $\Delta$ : $X^{X} \rightarrow X^{X} \times X^{X}$ inducing a co-algebra structure on $H_{*}\left(X^{X} ; Q\right)$ it follows that 
$H_{*}\left(X^{X} ; Q\right)$ is a Hopf algebra. We say that an element $\lambda \in H\left(X^{X} ; Q\right)$ is primitive if $\Delta_{*}(\lambda)=1 \otimes \lambda+\lambda \otimes 1$. The following result is essentially due to Gottlieb (1972).

Lemma 5.1. Let $\omega: X^{X} \rightarrow X$ be the evaluation map. Suppose that $H_{*}(X)$ is finitely generated. Let $\lambda \in H_{n}\left(X^{X} ; Q\right)$ be primitive and suppose that $\omega_{*}(\lambda) \neq 0$. Then if the Euler characteristic $\chi(X) \neq 0$, we have $\omega_{*}\left(\lambda^{k}\right) \neq 0$ for all $k>0$.

Now suppose that $f: A \rightarrow X$ is a map satisfying cat $f<2$, that is, we can find a map $\phi: A \rightarrow X \vee X$ such that $j \phi \simeq \Delta f$. It is then easily checked that for all $\alpha$ in $H_{*}(A ; Q), \Delta_{*} f_{*}(\alpha)=f_{*}(\alpha) \otimes 1+1 \otimes f_{*}(\alpha)$. In particular, this means that if $f$ is actually a map $A \rightarrow X^{X}$, we have that $f_{*}(\alpha)$ is primitive. Such would be the situation if $A$ were a co- $H$-space, for we might let $\phi=\psi f$ where $\psi$ is the co- $H$-structure on $A$.

Theorem 5.2. Suppose $f: A \rightarrow X$ is cyclic where $A$ is a co-H-space. Suppose $A$ is a finite dimensional $C W$-complex, and $H_{*}(X)$ and $\pi_{*}(X)$ are finitely generated. If $\chi(X) \neq 0$, then $\Sigma f$ is an element of finite order in $[\Sigma A, \Sigma X]$.

Proof. Since $f$ is cyclic, we can find a map $g: A \rightarrow X^{X}$ such that $\omega g \simeq f$. Now since $A$ is a co- $H$-space, it follows that cat $g<2$, and hence $g_{*}(\alpha) \in H_{*}\left(X^{X} ; Q\right)$ is primitive for all $\alpha$ in $H_{*}(A ; Q)$. We claim that $f_{*}=0: \tilde{H}_{*}(A ; Q) \rightarrow \tilde{H}_{*}(X ; Q)$. For if not, then we can find an element $\alpha \in H_{n}(A ; Q)$ for some $n>0$ such that $f_{*}(\alpha) \neq 0$. Since $g_{*}(\alpha)$ is primitive and $\omega_{*}\left(g_{*}(\alpha)\right)=f_{*}(\alpha) \neq 0$, it follows from Lemma 5.1 that $\omega_{*}\left(g_{*}(\alpha)^{k}\right) \neq 0$ for all $k>0$. According to Theorem 1 of Gottlieb (1972), we have $H_{*}(X ; Q) \cong \omega_{*}\left[g_{*}(\alpha)\right]_{\infty} \otimes M_{\infty}$ as vector spaces over $Q$, where $\left[g_{*}(\alpha)\right]_{\infty}$ is the subspace of $H_{*}\left(X^{X} ; Q\right)$ generated by $1, g_{*}(\alpha), g_{*}(\alpha)^{2}, \ldots$ if the dimension of $\alpha$ is even, and generated by $1, g_{*}(\alpha)$ if the dimension of $\alpha$ is odd, and $M_{\infty}$ denotes the elements of $H_{*}(X ; Q)$ of depth zero (see Gottlieb (1972) for details and definitions). If the dimension of $\alpha$ is even, this would contradict the fact that $H_{*}(X)$ is finitely generated, and if the dimension of $\alpha$ is odd, it would contradict the fact that $\chi(X) \neq 0$. Hence $f_{*}=0$. By duality, we have that $f^{*}=0: \tilde{H}^{*}(X ; Q) \rightarrow \tilde{H}^{*}(A ; Q)$. Now consider the map $h=e^{\prime} f: A \rightarrow X$ $\rightarrow \Omega \Sigma X$ where $e^{\prime}: X \rightarrow \Omega \Sigma X$ is the usual map. Then $h^{*}=f^{*} e^{*}=0$ : $\tilde{H}^{*}(\Omega \Sigma X ; Q) \rightarrow \tilde{H}^{*}(A ; Q)$. According to Arkowitz and Curjel (1964), it now follows that $h=e^{\prime} f$ is an element of finite order in $[A, \Omega \Sigma X]$. Hence its adjoint $\Sigma f$ is an element of finite order in $[\Sigma A, \Sigma X]$. 
COROllary 5.3. Let $A$ be a co-H-space which is a CW-complex of dimension $<2 n-1$, and suppose that $X$ is $(n-1)$-connected. Suppose that $H_{*}(X)$ is finitely generated and $\chi(X) \neq 0$. If $f: A \rightarrow X$ is cyclic, then $f$ is an element of finite order in $[A, X]$.

Corollary 5.4. Suppose that $X$ is $(n-1)$-connected and $H_{*}(X)$ is finitely generated and $\chi(X) \neq 0$. If $f$ is an element of $G_{m}(X)$, we have that $\Sigma f$ is an element of finite order in $\pi_{m+1}(\Sigma X)$. In particular, if $m<2 n-1$, then $f$ is an element of finite order.

The following result is essentially due to Gottlieb (1972) (see Theorem 5). We state it here for co- $H$-spaces in general instead of for suspensions.

Lemma 5.5. Suppose $X$ is a co-H-space. If $\omega_{*}: \tilde{H}_{*}\left(X^{X} ; Q\right) \rightarrow \tilde{H}_{*}(X ; Q)$ is non-zero, then $X$ is a rational homology $n$-sphere for some odd integer $n$.

Corollary 5.6. Let $X$ be a co-H-space which is not a rational homology $n$-sphere, where $n$ is odd, and let $A$ be a finite dimensional $C W$-complex. If $f: A \rightarrow X$ is cyclic, then $\Sigma f$ is an element of finite order.

Proof. Under the hypotheses, we have $\omega_{*}=0$. Here since $f$ is cyclic, we have $f_{*}=0$. The rest of the proof goes along the same lines as the arguments in the proof of Theorem 5.2.

Similarly, we have the following result.

THEOREM 5.7. Let $A$ be a finite dimensional $C W$-complex and let $X$ be a space such that $\Sigma X$ is not a rational homology $n$-sphere, $n$ odd. Let $f: A \rightarrow X$ be a map. If $\sum f$ is cyclic, then it is of finite order.

COROllary 5.8. Let $f: A \rightarrow X$ be a map, where $X$ is a homotopy associative $H$-space and $A$ is a finite dimensional $C W$-complex. If $\Sigma f$ is cyclic then $f$ is an element of finite order.

Proof. According to the hypotheses, it follows that $\Sigma X$ is not a rational homology $n$-sphere, $n$ odd. Since $\Sigma f$ is cyclic, by Theorem 5.7 , it follows that $\Sigma f$ is an element of finite order. Taking adjoints, we see that $e^{\prime} f$ is of finite order. Thus there exists a positive integer $k$ such that $k\left(e^{\prime} f\right)=0$. But since $X$ is a homotopy associative $H$-space, there exists an $H$-map $s^{\prime}: \Omega \Sigma X \rightarrow X$ such that $s^{\prime} e^{\prime} \simeq 1_{X}$. Thus $0=s^{\prime} k\left(e^{\prime} f\right)=k s^{\prime} e^{\prime} f=k f$ and hence $f$ is of finite order. 
Remark. The conditions that $H_{*}(X)$ and $\pi_{*}(X)$ be finitely generated might imply that $X$ is contractible. This would be the case if $X$ were required to be a finite compact complex.

\section{Acknowledgement}

The author is indebted to Professor C. S. Hoo for his useful suggestions and assistance during the preparation of the paper.

\section{References}

M. Arkowitz and C. R. Curjel (1964), Groups of homotopy classes (Lecture Notes in Mathematics, Vol. 4, Springer-Verlag).

M. Arkowitz and C. R. Curjel (1967), 'On maps of $H$-spaces' Topology 6, 137-148.

T. Ganea (1967), 'Induced fibrations and cofibrations', Trans. Amer. Math. Soc. 127, 442-459.

T. Ganea (1968), 'Cyclic homotopies', Illinois J. Math. 12, 1-4.

D. H. Gottlieb (1965), 'A certain subgroup of the fundamental group', Amer. J. Math. 87, 840-856.

D. H. Gottlieb (1969), 'Evaluation subgroups of homotopy groups', Amer. J. Math. 91, 729-756.

D. H. Gottlieb (1972), 'The evaluation map and homology', Michigan Math. J. 19, 289-297.

C. S. Hoo (1972), 'Cyclic maps from suspensions to suspensions', Canad. J. Math. 24, 789-791.

S. T. Hu (1959), Homotopy theory (Academic Press, New York).

G. E. Lang Jr. (1970), Evaluation subgroups and related topics (Ph. D. dissertation, Purdue Univ.).

K. Varadarajan (1969), 'Generalized Gottlieb groups', J. Indian Math. Soc. 33, 141-164.

Department of Economics and Statistics

National University of Singapore

Singapore 